Marcelo Lacerda de Oliveira

\title{
Análise da cadeia de suprimentos de importação de partes e peças automotivas envolvendo os conceitos de produção Completely-Knocked-Down (CKD) e Part-By-Part (PBP)
}

Dissertação de Mestrado (Opção profissional)

Dissertação apresentada como requisito parcial para obtenção do grau de Mestre pelo Programa de PósGraduação em Logística do Departamento de Engenharia Industrial do Centro Técnico Científico da PUC-Rio.

Orientador: Luiz Felipe Roris Rodriguez Scavarda do Carmo 


\section{Análise da cadeia de suprimentos de importação de partes e peças automotivas envolvendo os conceitos de produção Completely-Knocked-Down (CKD) e Part-By-Part (PBP)}

Dissertação apresentada como requisito parcial para obtenção do grau de Mestre pelo Programa de Pós-Graduação em Logística do Departamento de Engenharia Industrial do Centro Técnico Científico da PUC-Rio. Aprovada pela Comissão Examinadora abaixo assinada.

Prof. Luiz Felipe Roris Rodriguez Scavarda do Carmo

Orientador

Departamento de Engenharia Industrial - PUC-Rio

Prof. Nélio Domingues Pizzolato

Departamento de Engenharia Industrial - PUC-Rio

Prof. Edson José Dalto

Veris Educacional S/A, IBMEC Rio de Janeiro

Prof. José Eugênio Leal

Coordenador(a) Setorial do Centro Técnico Científico - PUC-Rio 
Todos os direitos reservados. É proibida a reprodução total ou parcial do trabalho sem autorização da universidade, do autor e do orientador.

\section{Marcelo Lacerda de Oliveira}

Graduou-se em Economia na Faculdade de Economia e Administração da Universidade Federal de Juiz de Fora em 1999. Pós-graduou-se em Logística Industrial pela Universidade Federal de Juiz de Fora em 2001. Atualmente trabalha na coordenação de operações logísticas da Empresa ALB Automotive Logistics.

Ficha Catalográfica

Oliveira, Marcelo Lacerda de

Análise da cadeia de suprimentos de importação de partes e peças automotivas envolvendo os conceitos de produção Completely-Knocked-Down (CKD) e Part-By-Part (PBP) / Marcelo Lacerda de Oliveira ; orientador: Luiz Felipe Roriz Rodriguez Scavarda do Carmo. - 2007.

123 f.: il.; $30 \mathrm{~cm}$

Dissertação (Mestrado em Engenharia Industrial) Pontifícia Universidade Católica do Rio de Janeiro, Rio de Janeiro, 2007.

Inclui bibliografia.

1. Engenharia Industrial - Teses. 2. Análise da cadeia de suprimentos de importação de partes e peças automotivas. 3. Completely-Knocked-Down (CKD) 4. PartBy-Part (PBP). 5. Logística. 6. Supply Chain Management. I. Carmo, Luiz Felipe R. R. Scavarda do. II. Pontifícia Universidade Católica do Rio de Janeiro. Departamento de Engenharia Industrial. III. Título. 


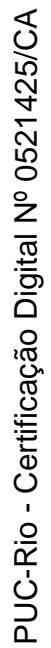

Para minha esposa e minha família. 


\section{Agradecimentos}

A minha esposa, Larissa, por todo incentivo, compreensão, apoio e principalmente pelo amor e carinho.

Ao meu Orientador Professor Luiz Felipe Scavarda, pela atenção dada, pela dedicação e pelo interesse na realização deste trabalho.

Aos meus pais, irmãos, familiares e amigos pelo apoio. 


\section{Resumo}

Oliveira, Marcelo Lacerda de; Carmo, Luiz Felipe R. R. Scavarda do.

Análise da cadeia de suprimentos de importação de partes e peças automotivas envolvendo os conceitos de produção CompletelyKnocked-Down (CKD) e Part-By-Part (PBP). Rio de Janeiro, 2007. 123p. Dissertação de Mestrado (Opção profissional) - Departamento de Engenharia Industrial, Pontifícia Universidade Católica do Rio de Janeiro.

O fluxo intenso de mercadorias entre países é uma característica marcante da globalização. Isto ocorre porque cada vez mais empresas procuram instalar unidades produtivas próximas aos mercados consumidores, enquanto que seus fornecedores situam-se em vários países. Conseqüentemente, cresce a importância do planejamento da movimentação de materiais dentro de um sistema logístico internacional. Decisões relativas à escolha dos meios de transportes, dos terminais multi-modais de carga, dos equipamentos para manuseio, das estratégias de armazenagem, da embalagem e unitização dos produtos, e de sistemas de informação, são estratégicas para o sucesso do projeto de rede. As cadeias de suprimentos projetadas pelas multinacionais automotivas são um dos os maiores exemplos de redes de fluxos internacionais de produtos. Neste contexto, a presente dissertação realiza uma análise da logística de importação de partes e peças amparadas pelos conceitos de produção, embalagem e transporte Completely-Knocked-Down (CKD) e Part-ByPart (PBP), através do estudo de caso dos projetos dos automóveis Classe A e Classe C, produzidos pela montadora DaimlerChrysler do Brasil na sua unidade produtiva localizada na cidade de Juiz de Fora.

\section{Palavras-chave}

Completely-Knocked-Down; Part-By-Part, Supply Chain Management, Logística; Embalagem; Armazenagem; Transporte; Sistemas de Informação; Desembaraço Aduaneiro. 


\section{Abstract}

Oliveira, Marcelo Lacerda de; Carmo, Luiz Felipe R. R. Scavarda do (Advisor). Supply chain analysis of automotive import parts involving Completely-Knocked-Down (CKD) and Part-By-Part (PBP) production concepts. Rio de Janeiro, 2007. 123p. M.Sc. Dissertation (Professional option) - Departamento de Engenharia Industrial, Pontifícia Universidade Católica do Rio de Janeiro.

The increase of material flows between countries is a strong characteristic of globalization. It happens because more and more companies are installing production units near their main markets and their suppliers' plants can be in located in different sites around the world. Therefore the importance of supply chain planning increases into the logistics system. Decisions about warehousing, transport modes, inter-modal terminals, handling equipments, packaging, unitization and information systems are strategic for the success of the network project. The multinational automakers are one of the biggest players of the international material flow. Within this context, the present master's thesis proposes a supply chain analysis of automotive import parts involving Completely-Knocked-Down (CKD) and Part-By-Part (PBP) production, packaging and transport concepts, by the analysis of a case study of A-Class and C-Class logistics project models assembled by DaimlerChrysler in Juiz de Fora city (Brazil).

\section{Keywords}

Completely-Knocked-Down; Part-By-Part; Supply Chain Management; Logistic; Packaging; Warehousing; Transport; Information Systems; Customs Clearance. 


\section{Sumário}

1 Introdução 15

1.1. Objetivos da pesquisa 15

1.2. Delimitação do escopo da dissertação 16

1.3. Metodologia científica 16

$\begin{array}{ll}\text { 1.4. Estrutura da dissertação } & 17\end{array}$

2 Revisão Bibliográfica $\quad 18$

$\begin{array}{ll}\text { 2.1. Projeto de rede } & 18\end{array}$

2.2. Embalagem 19

2.2.1. Conceito, função e classificação da embalagem 20

2.2.2. Padronização e unitização 23

2.3. Armazenagem 24

2.3.1. Movimentação de materiais $\quad 25$

2.3.2. Fatores importantes para a eficiência de um armazém 26

2.3.3. Atividade de Picking 28

2.3.4. Funcionalidades da armazenagem 31

2.3.5. Alternativas de armazenagem 31

2.4. Transporte 33

2.4.1. Classificação dos modais de transporte 33

2.4.2. Intermodalidade e multimodalidade 38

2.5. Just-in-time 40

2.5.1. Kanban 41

2.6. CBU, SKD, CKD e PBP 42

2.6.1. Concepção e fatores determinantes 43

2.6.2. Características operacionais 44

2.7. Informação e sistemas 46

2.7.1. EDI (Electronic data interchange) 47

2.7.2. Warehouse Management System (WMS) 48

2.8. Desembaraço aduaneiro 51

2.8.1. Licenciamento de importação (LI) e declaração de importação (DI) 52

2.8.2. Sistema Integrado de Comércio Exterior (Siscomex) 53 
3 Projeto 1

3.1. Descrição da operação 58

3.1.1. Centro de consolidação 58

3.1.2. Trânsito da Alemanha para o Brasil 59

3.1.3. Porto Seco 61

3.1.4. Armazém Geral 64

3.2. Considerações complementares sobre embalagem, armazenagem, movimentação e transporte 66

3.3. Considerações complementares sobre documentação, informação e sistemas $\quad 69$

3.3.1. Análise da necessidade de material 73

3.4. Considerações complementares sobre desembaraço aduaneiro 74

4 Projeto $2 \quad 75$

4.1. Descrição da operação $\quad 76$

4.1.1. Processos de robbing 76

4.1.2. Processo de reposição das embalagens e entrega dos conjuntos $\quad 80$

4.2. Considerações complementares sobre embalagem, armazenagem, $\begin{array}{ll}\text { movimentação e transporte } & 81\end{array}$

4.3. Considerações complementares sobre documentação, informação e sistemas $\quad 85$

4.4. Considerações complementares sobre desembaraço aduaneiro 88

5 Projeto $3 \quad 90$

$\begin{array}{ll}\text { 5.1. Descrição da operação } & 91\end{array}$

5.2. Considerações complementares sobre embalagem, armazenagem, $\begin{array}{ll}\text { movimentação e transporte } & 91\end{array}$

5.3. Considerações complementares sobre documentação, informação e $\begin{array}{ll}\text { sistemas } & 93\end{array}$

5.4. Considerações complementares sobre desembaraço aduaneiro 95

6 Conclusão 97

6.1. Os conceitos CBU, SKD, CKD e PBP $\quad 97$

6.2. Integração entre o fluxo de materiais e o fluxo de informação 100 
6.3. O processo de peças de reposição do CKD 103

6.4. Recomendações para trabalhos futuros 103

7 Referências Bibliográficas $\quad 105$

Anexo I: Fábrica da DaimlerChysler do Brasil em Juiz de Fora 115

$\begin{array}{ll}\text { Anexo II: Mapa da região sudeste } & 116\end{array}$

Anexo III: Porto do Rio de Janeiro - Tecon 2

Anexo IV: Porto Seco e Armazém Geral de Juiz de Fora 118

Anexo V: Planta baixa do armazéns KLT e GLT - Projeto 1

Anexo VI: Embalagens e estrutura do armazém GLT - Projeto 1

Anexo VII: Embalagens e estrutura do armazém KLT - Projeto 1

Anexo VIII: Embalagens e estrutura do armazém - Projeto 2

Anexo IX: Mapa de armazenagem - Projeto 2 


\section{Lista de figuras}

Figura 1: Cadeia de suprimento - Projeto $1 \quad 57$

Figura 2: Etiqueta de identificação das caixas - Projeto $1 \quad 68$

Figura 3: Fluxo principal de documentação e informação - Projeto 1

Figura 4: Tracking \& Tracing SAP R/3 - Projeto 1

Figura 5: Cadeia de suprimentos - Projeto 2

Figura 6: Etiqueta de identificação dos paletes - Projeto 2

Figura 7: Fluxo principal de documentação e informação - Projeto $2 \quad 87$

Figura 8: Tracking \& Tracing SAP R/3 - Projeto $2 \quad 88$

Figura 9: Cadeia de suprimento - Projeto $3 \quad 90$

Figura 10: Fluxo principal de documentação e informação - Projeto $3 \quad 94$

Figura 11: Tracking \& Tracing - Projeto $3 \quad 95$

Figura 12: Conceitos logísticos - CBU, SKD, CKD e PBP. 98 


\section{Lista de tabelas}

Tabela 1: Alternativas de sistema de armazenagem (Banzato, 2001c) 27

Tabela 2: Estratégias de pickings (Rodrigues, 1999) 30

Tabela 3: Classificação das características operacionais por modal de transporte (Bowersox \& Closs, 2001) 34

Tabela 4: Interfaces principais - Projeto 1

Tabela 5: Interfaces principais - Projeto 2

Tabela 6: Características básicas dos conceitos SKD, CKD e PBP 98

Tabela 7: Software e hardware usados na operação no Brasil 101 


\section{Siglas}

AG - Armazém Geral

ASN - Advanced Shipment Notification

ASRS - Sistemas Automatizados de Armazenagem e Recuperação

AWB - airwaybill

BACEN - Banco Central do Brasil

$\mathrm{BL}$ - Bill of Lading

$\mathrm{CBU}$ - Completely-Build-Up

CC - Centro de Consolidação

$\mathrm{Cl}$ - Comprovante de Importação

CKD - Completely-Knocked-Down

CTRC - Conhecimento de Transporte Rodoviário de Carga

DI - Declaração de Importação

DRP - Distribution Requirements Planning

DTA - Documento de Trânsito Aduaneiro

EADI - Estação Aduaneira Interior

EDI - Eletronic Data Interchange

EDIFACT - EDI for Administration, Commerce and Transport

ETA - Estimated Time of Arrived

ETD - Estimated Time of Departure

FIFO - First-In-First-Out

FROB - Finalização do Robbing

GLT - Großladungsträger

GPS - Global Positioning System

ICMS - Imposto sobre Circulação de Mercadorias e Serviços

JIS - Just-In-Sequence

JIT - Just-In-Time

KLT - Kleinladungsträger

LCM - Lista Crítica de Material

LI - Licenciamento de Importação

LT - Lista de Transporte

MAA - Ministério da Agricultura

NCM - Nomenclatura Comum do Mercosul 


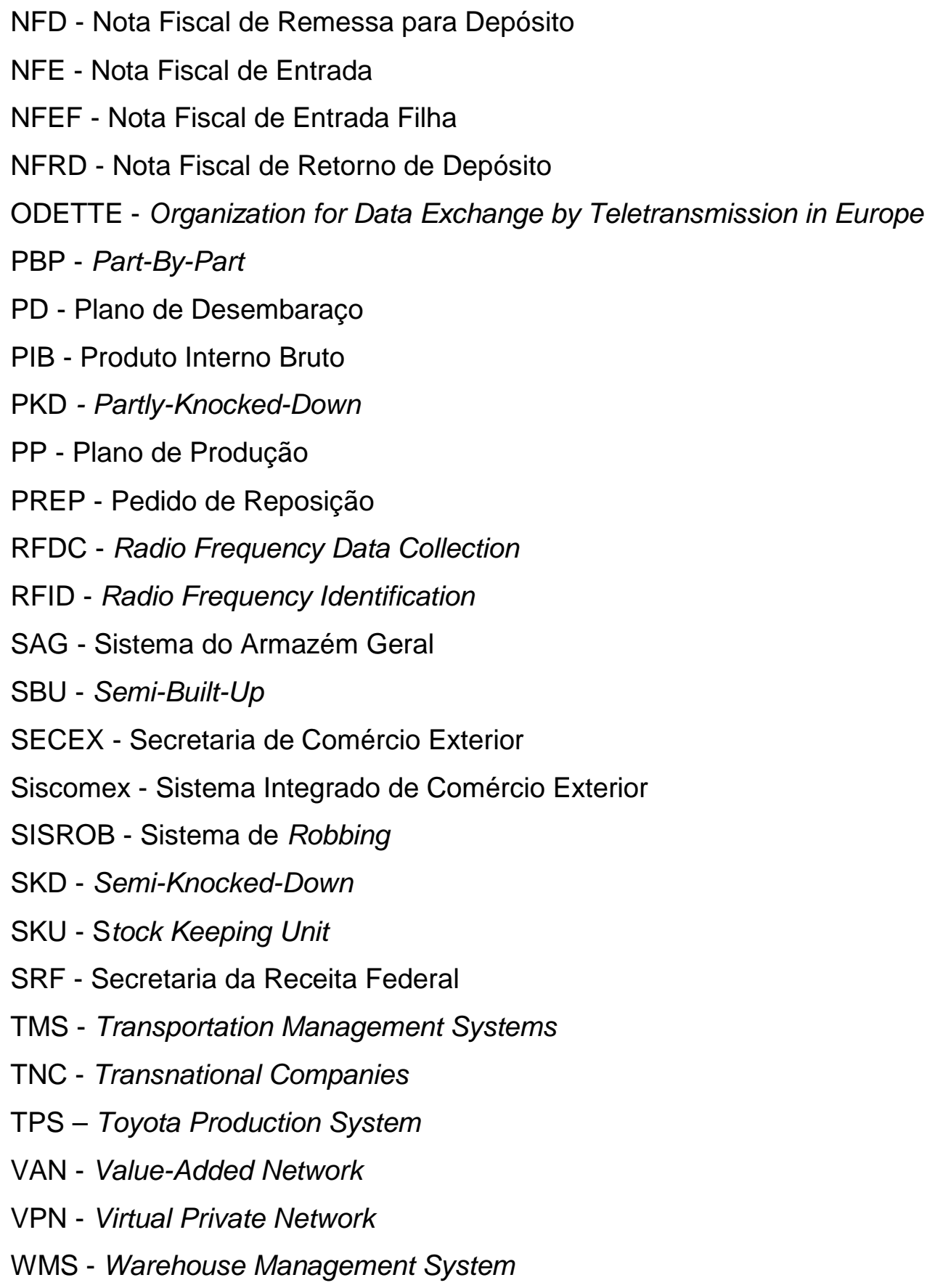

